\title{
Monte Carlo Study of Degenerate Behavior of AB Diblock Copolymer/Nanoparticle under
}

\section{Cylindrical Confinement}

Yingying Wang ${ }^{\dagger \neq, \S}$, Yuanyuan Han* ${ }^{* \dagger}$, Jie Cui ${ }^{*},{ }^{\dagger}$, Wei Jiang ${ }^{\dagger}$, Yingchun Sun ${ }^{\star}$

${ }^{\dagger}$ State Key Laboratory of Polymer Physics and Chemistry, Changchun Institute of Applied Chemistry, Chinese Academy of Sciences, Changchun 130022, Jilin, China

${ }^{\star}$ Northeast Normal University, School of Physics, Changchun 130024, Jilin, China

${ }^{\S}$ University of Chinese Academy of Sciences, Beijing 100049, People's Republic of China

*Corresponding authors: *E-mail: yyhan@ciac.ac.cn (Yuanyuan Han); jcui@ciac.ac.cn (Jie Cui).

Telephone: 86-431-85262642. Fax: 86-431-85262126. 


\section{Supporting Information}

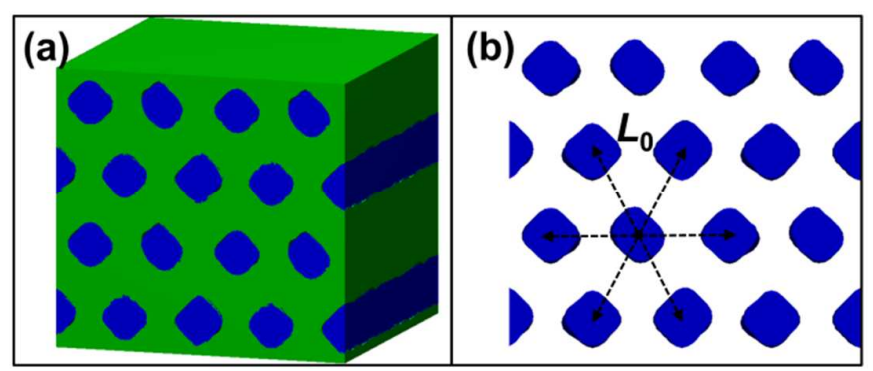

Figure S1. Typical hexagonally packed cylindrical phase formed by $\mathrm{A}_{5} \mathrm{~B}_{15}$ diblock copolymers in bulk (a). For the purpose of clarity, only minor component $\mathrm{A}$ is drawn in blue (b). The minority block A and majority block B are represented by blue and green, respectively.
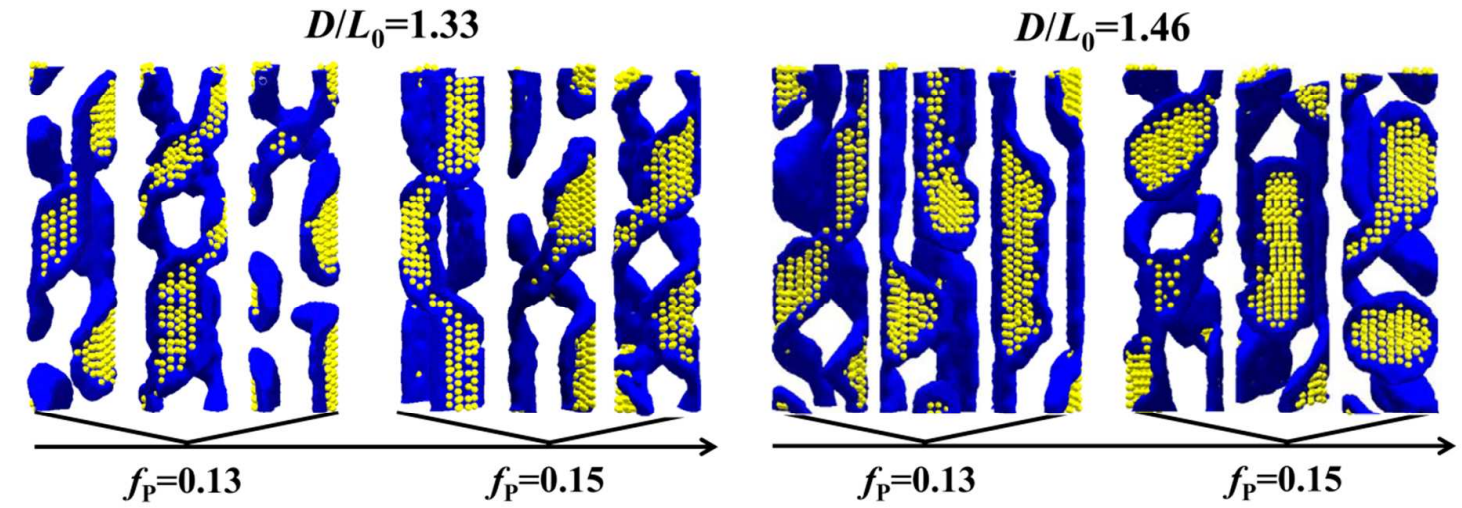

Figure S2. Typical disordered structures formed by $\mathrm{A}_{5} \mathrm{~B}_{15}$ diblock copolymer/NP mixture in cylindrical pores with different pore sizes $\left(D / L_{0}\right)$ and NP contents $\left(f_{P}\right)$. For the purpose of clarity, only minor component A and NPs are drawn. The minority block A and NPs are represented by blue and yellow, respectively. 
(a)

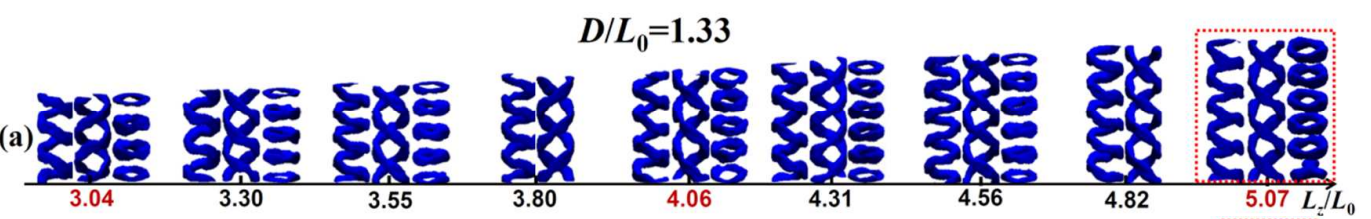

(b)
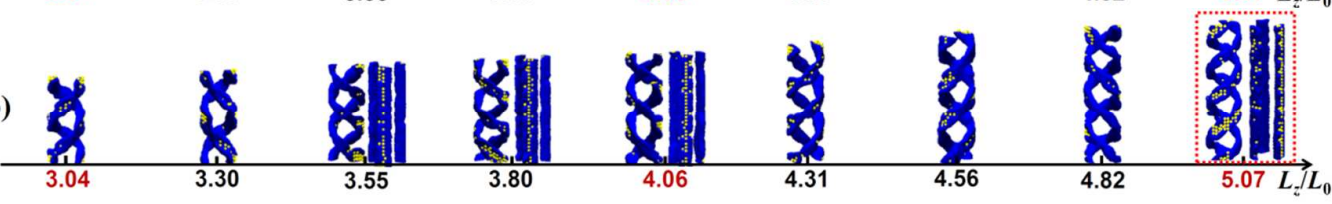

(c)

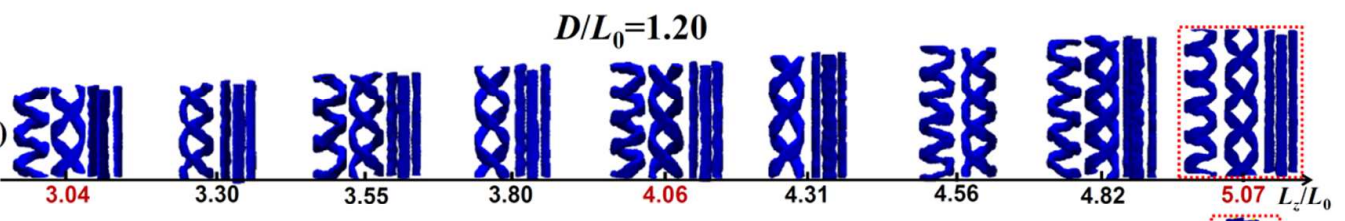

(d)

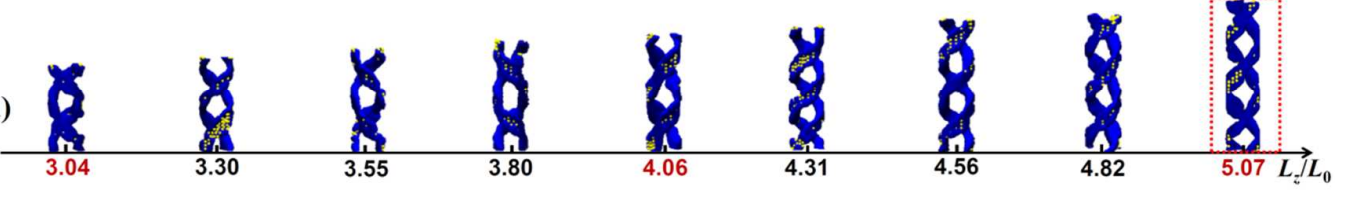

Figure S3. Nanostructures self-assembled by BCP/NP mixture in cylindrical pores as a function of the pore length $\left(L_{z} / L_{0}\right)$. The pore diameters and NP contents are (a) $D / L_{0}=1.33, f_{P}=0$; (b) $D / L_{0}=1.33, f_{P}=0.05 ;$ (c) $D / L_{0}=1.20, f_{P}=0 ;$ (d) $D / L_{0}=1.20, f_{P}=0.05$. The nanostructures obtained at $L_{z}=80\left(L_{z} / L_{0}=5.07\right)$ (Figure 2 in the manuscript) are also given for the purpose of comparison, which are marked with red boxes. Only the minority blocks A and NPs are drawn for clarity.

Table S1. Average interactive enthalpy densities of double helices under different pore lengths $\left(L_{z} / L_{0}\right)$ and NP contents $\left(f_{P}\right)$. The pore size is fixed at $D / L_{0}=1.20$.

\begin{tabular}{cccccccccc}
\hline \multirow{8}{*}{$\begin{array}{c}\text { PP content } \\
\left(f_{P}\right)\end{array}$} & $\mathbf{3 . 0 4}$ & $\mathbf{3 . 3 3}$ & $\mathbf{3 . 5 5}$ & $\mathbf{3 . 8 0}$ & $\mathbf{4 . 0 6}$ & $\mathbf{4 . 3 1}$ & $\mathbf{4 . 5 6}$ & $\mathbf{4 . 8 2}$ & $\mathbf{5 . 0 7}$ \\
\cline { 2 - 10 } & 1.029 & 1.032 & 1.031 & 1.032 & 1.031 & 1.030 & 1.034 & 1.030 & 1.030 \\
$\mathbf{0 . 0 0}$ & 1.024 & 1.024 & 1.025 & 1.030 & 1.028 & 1.027 & 1.035 & 1.026 & 1.026 \\
$\mathbf{0 . 0 5}$ & & & & & & & &
\end{tabular}


Table S2. Average interactive enthalpy densities of double helices under different pore lengths $\left(L_{z} / L_{0}\right)$ and NP contents $\left(f_{P}\right)$. The pore size is fixed at $D / L_{0}=1.33$.

\begin{tabular}{|c|c|c|c|c|c|c|c|c|c|}
\hline \multirow{2}{*}{$\begin{array}{l}\text { NP content } \\
\quad\left(f_{P}\right)\end{array}$} & \multicolumn{9}{|c|}{$\begin{array}{c}\text { Pore length } \\
\left(L_{t} L_{0}\right)\end{array}$} \\
\hline & 3.04 & 3.33 & 3.55 & 3.80 & 4.06 & 4.31 & 4.56 & 4.82 & 5.07 \\
\hline 0.00 & 1.037 & 1.026 & 1.026 & 1.015 & 1.021 & 1.023 & 1.029 & 1.026 & 1.032 \\
\hline 0.05 & 1.025 & 1.020 & 1.028 & 1.022 & 1.027 & 1.025 & 1.020 & 1.021 & 1.025 \\
\hline
\end{tabular}
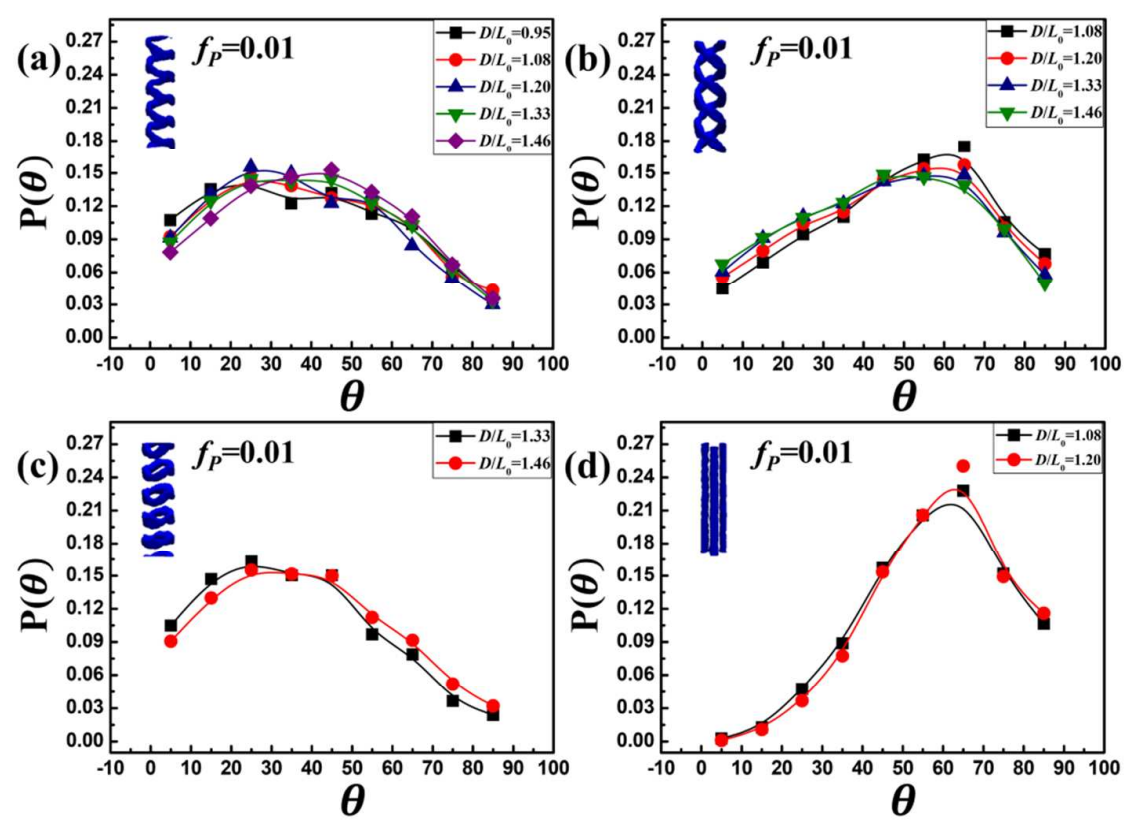

Figure S4. Distribution curves of the orientation angle $\theta$ for different degenerate structures obtained under different pore sizes in the case of $f_{P}=0.01$. 

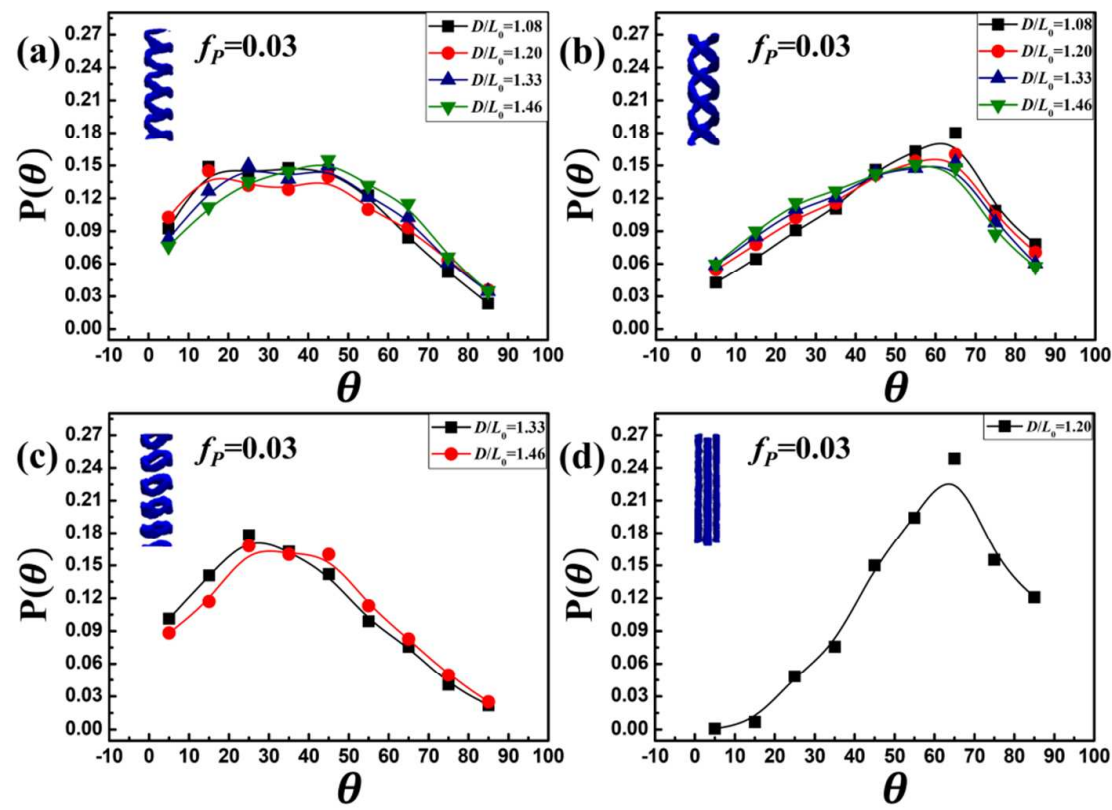

Figure S5. Distribution curves of the orientation angle $\theta$ for different degenerate structures obtained under different pore sizes in the case of $f_{P}=0.03$.
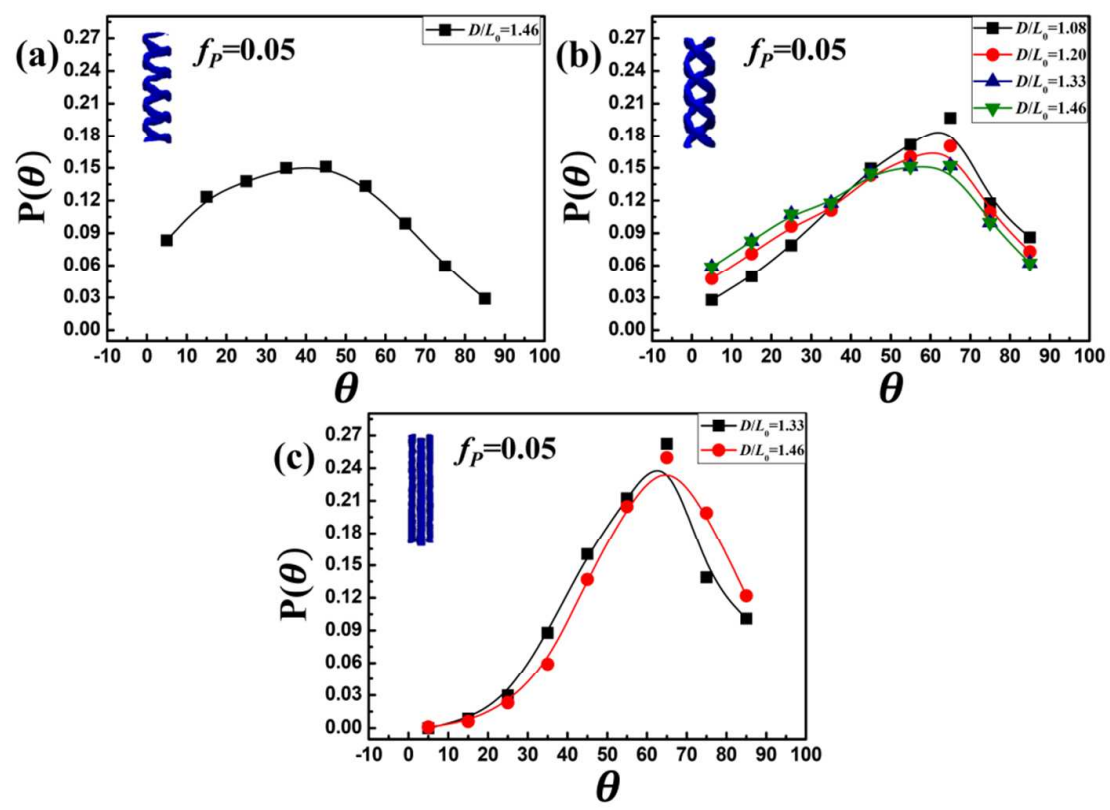

Figure S6. Distribution curves of the orientation angle $\theta$ for different degenerate structures obtained under different pore sizes in the case of $f_{P}=0.05$. 

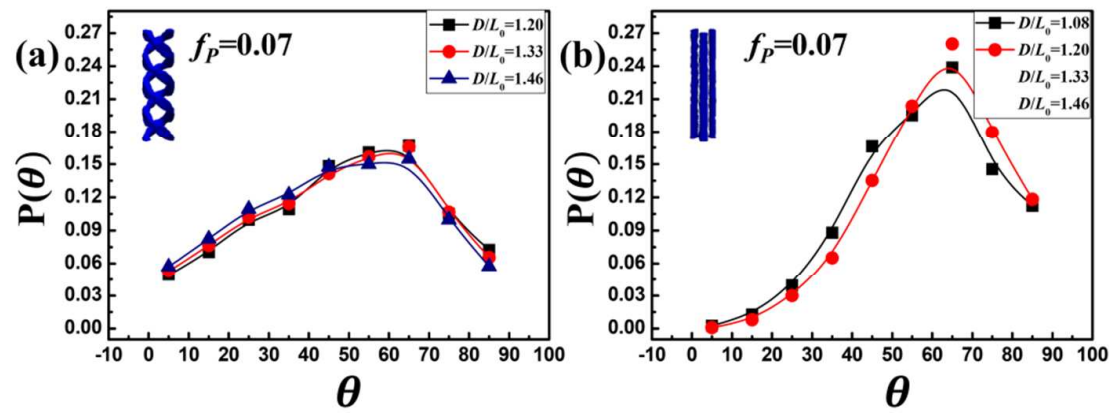

Figure S7. Distribution curves of the orientation angle $\theta$ for different degenerate structures obtained under different pore sizes in the case of $f_{P}=0.07$.
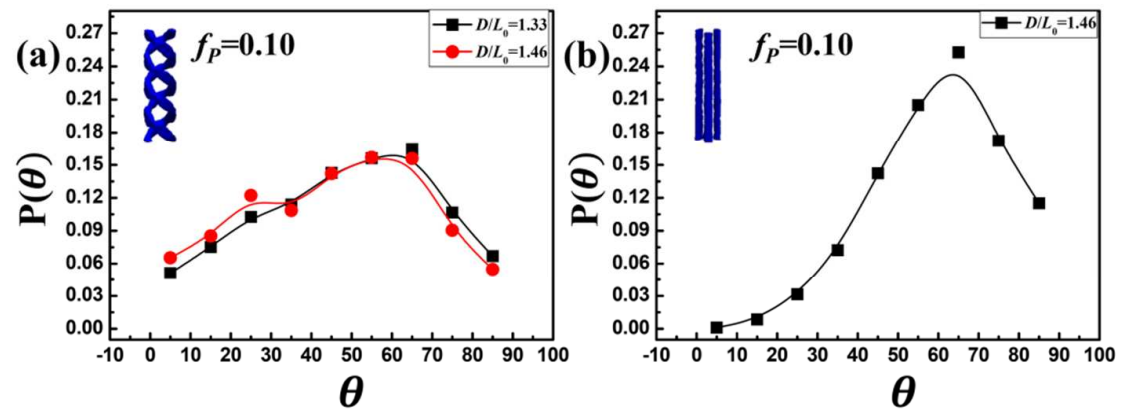

Figure S8. Distribution curves of the orientation angle $\theta$ for different degenerate structures obtained under different pore sizes in the case of $f_{P}=0.10$. 